\title{
APPLYING A COGNITIVE ARCHITECTURE TO CONTROL OF VIRTUAL NON-PLAYER CHARACTERS
}

\author{
Christopher McCollum \\ Charles Barba \\ Thomas Santarelli \\ 1035 Virginia Drive \\ Suite 300 \\ CHI Systems, Inc. \\ Fort Washington, PA 19034, U.S.A
}

\author{
John Deaton \\ 12000 Research Parkway \\ Suite 120 \\ CHI Systems, Inc. \\ Orlando, FL 32826, U.S.A
}

\begin{abstract}
CHI Systems, under contract to the U. S. Army Research Institute, is developing an immersive training system, called Virtual Environment Cultural Training for Operational Readiness (VECTOR), which applies highly experiential, scenario-based virtual environments to training in cultural familiarization. To produce an interactive, realistic training environment, the simulation must incorporate synthetic actors, or non-player characters (NPC's), that are capable of evaluating and responding to the cultural propriety of trainee's actions. The main focus of the paper is on explaining how iGEN ${ }^{\mathrm{TM}}$ cognitive modeling architecture is being used to create executable cognitive models and emotion models which inform and constrain the overall reactions and behaviors of NPC's toward the trainee. In addition to influencing the behavior of the active NPCs, the emotion models constrain interactions with NPC's encountered later in a scenario. In this way, the training system provides a means of modeling the overall cumulative emotional state of the simulated population.
\end{abstract}

\section{INTRODUCTION}

The skill with which the members of our military forces interact with members of other cultures is often critical for their survival and mission success. Cultural familiarization and understanding of group differences are especially critical to security forces that are responsible for managing potentially dangerous situations in foreign settings. CHI Systems is currently developing an immersive environment training system for the U.S. Army Research Institute (ARI) that will help forces engaged in Operations Other Than War (OOTW) acquire the ability to accurately assess and effectively respond to the motives and tendencies of indigenous populations. Specifically, the Virtual Environment Cultural Training for Operational Readiness (VECTOR) system that is being developed uses highly experiential, scenario-based training in virtual environments to teach culturally adaptive interaction skills related to military police and military intelligence tasks.

The virtual environment is designed to encourage a high degree of human interaction with the indigenous NPCs, as the trainee encounters prototypical social contexts and military situations. The effects of the trainee's actions, either positive or negative, are readily observable by the actions and affective state of NPCs within the game. The scenario reacts to these responses, and in the process, provides important information concerning the consequences of particular actions or omissions. The outcome of the simulation is, thus, not predetermined, but instead depends on human participants interpreting and reacting to the evolving scenario. The scenario represents a collection of synthetic actors, or non-player characters (NPC's), in the cultural environment that have predefined roles, tasks, and motivations. Some external events happen at fixed times, while others happen on the basis of proximity or other contingencies. Essentially, however, the scenario plays out solely on the basis of the trainee's actions in the environment. In this respect, it has much in common with current game technology. At the heart of this immersive environment are executable cognitive models and emotion models, which influence the overall reactions and behaviors of the NPC's toward the trainee. The ultimate goal is to produce a highly interactive, realistic virtual environment for cultural familiarization training.

The first part of the paper describes provides a brief overview of the specific training scenario that is being developed. The remainder addresses the role of cognitive and emotional models in informing and constraining the dynamic interactions between trainee and NPC's.

\section{VECTOR CULTURAL TRAINING}

To construct a realistic immersive environment, we gathered a large data set consisting of Arabic, as well as Kurd-specific, cultural information, as it applies to peace-keeping type mis- 
sions. This information was synthesized from multiple sources, including books (e.g., Izady 1992; Kreyenbroek \& Allison 1996), articles (e.g., Ruff 1998), and military technical reports and pamphlets (e.g., Wise et al. 1998). Some of the major cultural dimensions that U.S. forces would be required to understand in Kurdistan, as well as many other cultures, include: gender; religion; status; perceptions of/attitudes toward American culture/individuals interpersonal space (proxemics) and interaction; and emotion/personality tendencies or predispositions. A number of elements from each of these cultural dimensions are being integrated into the scenario.

Figure 1 below shows a sample screen-dump of the VECTOR display interface and the 5 major GUI components, each marked with a numbered symbol, including a General Status Display, NPC Name Placard, Mission Objectives List, Dialog Interaction Display, and a Mission Map. Each of these is explained below.;

1. General Status Display: This is used to display Mission Objective (MO) information, including MO changes, additions, and completion status, as well as each instance of a cultural rule violation oradherence. Additionally, at the end of each mis sion, a summary of the mission is displayed with respect to a review of each cultural rule that was measured.
2. NPC Placard: Each NPC's name, and their overall emotional state, is display above their avatar and is always parallel to the player's field of view. By default, the NPC emotional state for each NPC is not displayed until initial dialog interactions Those NPC's that don't have a name placard above there head are non-interactive and will not engage the user in dialog.

3. Mission Objectives Summary: This display toggle is used to view the current list of MOs and their status. Those MOs with a leading '-' character are not yet complete, those with a leading ' $\mathrm{X}$ ' are completed. The Mission Objectives Summary can be viewed at any time by holding down the 'Tab' key.

4. Dialog Interactions: This display is used to present a text version of the utterances that an NPC generates (this is in parallel to the '.wav' file that is played as a companion modality to present theauditory version of the NPC utterance). Additionally, the user can 'speak' to an NPC by mov ing the dialog selection cursor, indicated by the ' $>$ ' character and hitting return to send the utterance to the NPC that is being spoken to.

5. Scenario Map: This displays a 2-D overhead map of the current scenario and depicts the location of the active scenario objective.

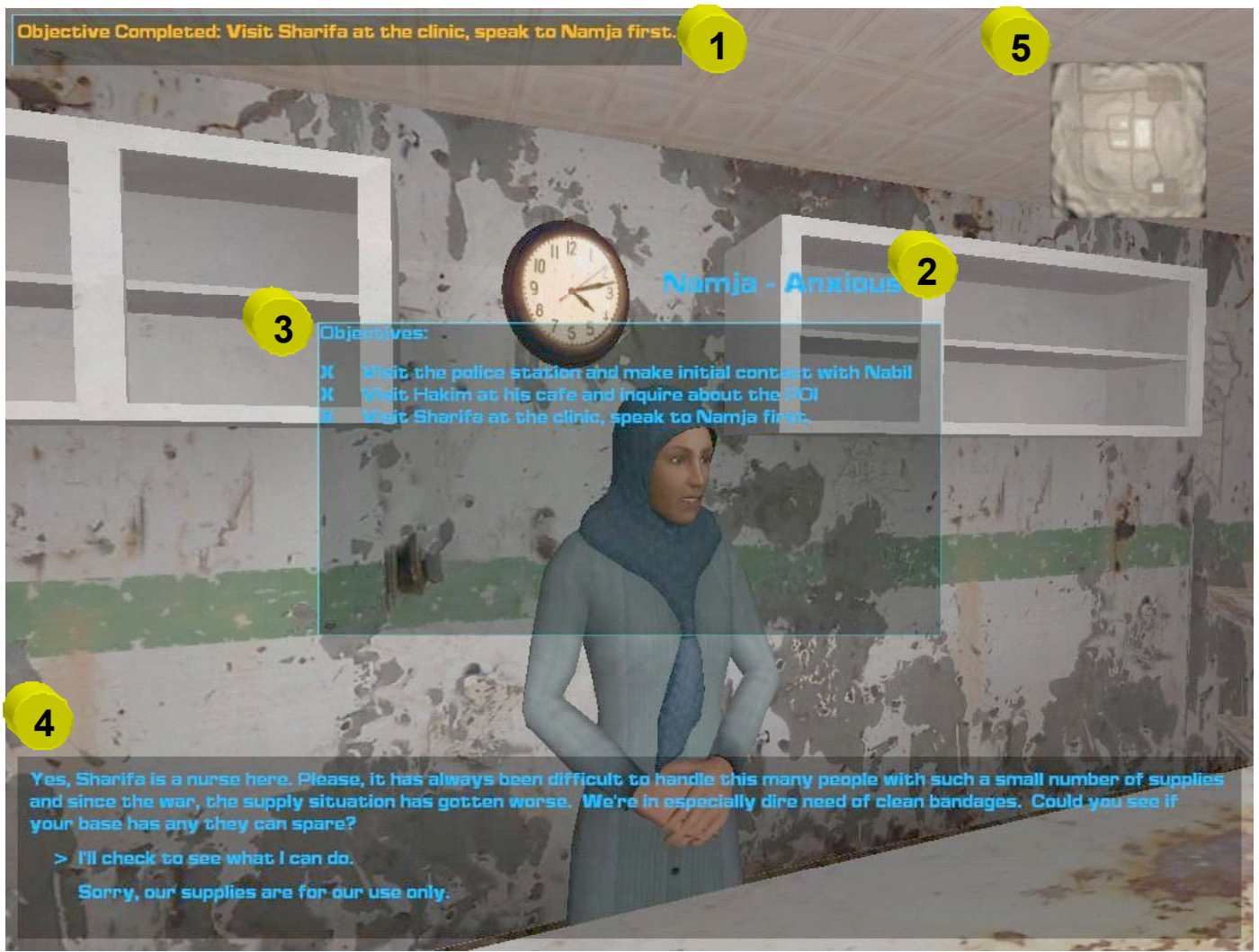

Figure 1: Sample Screenshot of Vector Display Interface 
The training scenario immerses the Trainee, represented as Squad Leader, in a virtual village and requires them to interact with various Indigenous Entities in order to gain information on the whereabouts of several Persons of Interest. The Trainee is free to move about the virtual village at will, as well as select the order of Locations he or she visits and the Indigenous Entities that he or she will engage. While moving through the village, the Trainee gathers information from Indigenous Entities as to the whereabouts of the Persons of Interest. Success in acquiring this information depends on the degree to which the Trainee demonstrates appropriate cultural knowledge and skills.

In most situations, the visual simulation has the firstperson perspective of the Trainee, and, thus, the Squad Leader is not visible in the visual scene. The Trainee moves through the virtual village using a mouse and keyboard. The Trainee can issue standing orders to the other Squad Members; however, the Squad Members behave in a semi-autonomous manner based on the Trainee's actions and according to a set of predefined military actions, behaviors, and rules of engagement. For example, when the Trainee moves forward, the Squad follows and maintains their formation and combat spacing. These Members include several enlisted personnel who provide specific functions (e.g., communications, medical, security). There is also an indigenous counterpart, a single synthetic entity, which is represented as an Iraqi police chief. The police chief functions as a trusted counterpart whose purpose is to facilitate the Trainee's mission by providing cultural advice when the trainee's actions are incorrect. All verbal communications are in English, in order to facilitate implementation of the initial scenario and increase interaction between the Trainee and Indigenous Entities (i.e., not mediated by Translator).

Major interaction sequences and cultural training opportunities are based on Location - e.g., police station, a café, several households, a marketplace, and a mosque. As the Trainee moves from one Location to another, the development of the overarching plotline - and thus Trainee success - depends on him or her avoiding behaviors that contradict cultural rules and demonstrating behaviors that are consistent with these rules. The former behaviors reduce the cooperativeness/tension of the Indigenous Entities (e.g., willingness to provide useful information), whereas the latter behaviors increase the cooperativeness/tension of these NPC's. The Indigenous Entities have varying, preset levels of cooperativeness/tension, which makes the Trainee's task of eliciting information more or less difficult from one location to the next. There are also plans to have changes in the cooperativeness/tension of currently engaged Indigenous Entities affect the pre-set levels of Indigenous Entities at other locations. This representation of "emotion contagion" is based on the idea that Indigenous Entities communicate with one another about the conduct of the Trainee.

\section{VECTOR TRAINEE INTERACTIONS}

In order for interactions to take place three basic elements are necessary - a Location, an Indigenous Entity, and the Trainee Entity. The Trainee Entity is the visual representation of the simulation user's presence and position within the virtual environment. The Trainee Entity is under the direct control of the user in terms of locomotion, selection of dia$\log$, selection of actions, and the choice to interact with all other simulation entities. To interact with NPC's, the user of the training system provides a GUI menu that allows the trainee to select dialog interactions through keyboard entry.

Interaction with Indigenous Entities can only occur in specific simulation Locations, which provide a stage for simulation events to unfold. This is necessary to provide predefined interaction opportunities, which contain variable outcomes and interaction evolutions based on user choices and the degree to which he or she can build trust in or otherwise negatively affect the Indigenous Entities present in the location. The two main types of locations are buildings and loiter points. Buildings can fall into several types of structures, including residences and specialized buildings (e.g., café, police station, and mosque). Interaction opportunities are based on the proximity of the Trainee Entity to Locations and Indigenous Entities.

The speech of NPC's is delivered by a WAV file output with concurrent text. An entity response (either an action or a dialog) is the direct result of an entity interaction, which evaluates the Trainee's actions and assesses its own internal and external state in order to generate a response from a set of possible responses. Only responses that are consistent with the entities' predisposition and allowable under the Location's present sub-plot position can be selected.

The user can interact with both the Squad Members and the Translator for assistance in conducting the military mission. These interactions can occur anywhere in the virtual world and do not require a designated Location. In order to directly interact with other simulation entities, the Translator must be instructed to do so by the user. Autonomous behavior of the Translator would reduce the trial and error value of the simulation. The Translator can, however, offer preemptive advice to the user in the case where a blatantly inappropriate cultural action is about to be taken by the user. The Translator Entity can also be queried by the user and can serve as an instructor agent for cultural information.

Squad Member Entities, and to a lesser extent the Translator Entity, functions in a semi-autonomous mode with respect to gross movements and actions. Squad Member Entities can, in effect, follow the Trainee Entity throughout the simulation and provide automatic context specific behaviors (e.g., forming a perimeter when the Trainee Entity approaches a door). Squad Members' actions are based on U.S. Army squad level movement doctrine.

Major processes underlying entity behaviors and memory include: response generation - what an entity will decide to do or say; advice generation - what the Translator 
may say in response to a query from the Trainee; interactive evaluation - how the Trainee's actions are scored; psychological state propagation - how interaction may affect the whole village; and training evaluation - how feedback is provided to the Trainee. Data stores consist mainly of actions and dialog alternatives, which represent all possible entity behaviors. Additional data stores include scenario related parameters and cultural rules. Transient data stores are used to represent behavioral state information for both individual entities and the overall village (i.e., the village mood).

\section{NPC BEHAVIOR AND AFFECT}

An important determinant of the success of the VECTOR training system is the representation of significant aspects of NPCs' affective processes, as well as their cultural background, in the scenario. According to our ontology of culture (see Triandis 1994; Strauss and Quinn 1997), interactions between individuals are governed by implicit norms and procedures, which are organized in individuals' minds as shared cognitive schemas. These schemas provide members of a culture with a complex and interactive set of principles and procedures (e.g., "cultural rules") ranging from coherent verbal interactions to acceptable social behaviors. In this way, shared schemas provide individuals with a distinct lens through which to see the world. Culture, then, acquires its motivational force - its power to instigate and shape behavior - by activating emotions within individuals. Observing and performing culturally consistent behaviors trigger positive emotions within individuals, whereas observing and performing culturally inconsistent behaviors trigger negative emotions. It is this fundamental principle of culture/emotion that underlies all actions of synthetic actors toward the trainee. Emotional modeling is, therefore, the linchpin in the integration of cultural factors into synthetic actors.

One of the innovative features of the VECTOR environment is the use of executable cognitive models that contain emotion models, which influence overall reactions and behaviors of NPC's toward the Trainee. Additionally, while the initial focus of VECTOR is on the Arab and Kurdish culture and sub-culture in particular, a more generic model of culture was seen as an important requirement.

Much research has been conducted on the development of more realistic emotional behaviors for intelligent virtual agents. VECTOR leverages this experimental research in order to produce entities that respond to the Trainee in a realistic way during an evolving and variable plot sequence. Implementation of behavior and affect within the training system has required the adoption of three major constructs an NPC cognitive model for behavioral interaction and dia$\log$, an underlying emotion model within the NPC cognitive model, and a scenario scripting language that allows for the specification of all aspects of NPC capability per scenario. We adopt a computational model of emotion based on the model proposed by Ortony, Clore, and Collins (Ortony et. al., 1988), commonly referred to as the OCC model. They categorize over 20 emotional variables with respect to a valanced mapping of reaction to objects, events, and actors.

The cognitive models that control the NPC behaviors are written using the commercially available version of $\mathrm{CHI}$ Systems' intelligent agent construction toolset, iGENTM. iGEN ${ }^{\mathrm{TM}}$ was developed specifically to construct executable models of human-computer interaction in real-time, multitasking environments (Zachary, Ryder, Ross, and Weiland 1992). iGENTM is based on a theoretical framework that includes a model of human information processing architecture, derived from multiple component theories, and a set of theoretically-derived principles of operation. The architecture and principles of operation together define an information processing mechanism that has been captured in a Blackboard Architecture for Task-Oriented Networks (BATON), a software simulation of human cognitive, perceptual, and motor mechanisms. In order to simulate human performance in a given domain, BATON must be provided with a body of knowledge that captures how complex problems (such as command and control problems) in the domain are acquired, internalized, reasoned about, and acted on. iGEN ${ }^{\mathrm{TM}}$ thus also includes a rich language for describing and formalizing the knowledge in a form that corresponds to the ways in which it is used by the various components of BATON (e.g., perception, problem-representation, reasoning, action). The basic information processing organization defines four separate types of knowledge that a person uses in complex work tasks -- sensory/perceptual knowledge (used by the sensory/perceptual process), declarative knowledge (used in the 'mental model' held in extended working memory), procedural knowledge (used by the cognitive process), and motor knowledge (used by the action process). Declarative knowledge is represented in a blackboard framework structure (Hayes-Roth 1985) and procedural knowledge is represented in a conditional goal-tree, loosely based on the GOMS notation first developed by Card, Moran and Newell (1983).

\subsection{NPC Behavioral Modeling}

As has been described, a capability is desired that can adapt generic NPC models and enable them to support training content for a specific cultures, as well as extend them to support "idiosyncratic" cultural features (ones that do not fit within the cultural template, but are still required for training) and instantiate them for individuals. The initial implementation takes the form of NPC profiles and scenario scripts. When the scenario script specifies instantiation of a specific actor (whose characteristics are drawn from a specific cultural profile), the model instance is created and reads the profile. The parameters of this profile are adjusted and extended by reading additional parameters specified with the actor definition within the scenario script. These additional parameters represent offsets from the norm represented by the cultural profile, as well as simple extensions that may be used by the cognitive model itself (such as name and gender 
of the actor). This scripting language allows for the construction of a canonical cognitive model of NPC interactions while allowing for infinite NPC character creation through the construction of new, or modifications of existing libraries, of NPC scenario scripts.

Based on our preliminary analysis we have identified a candidate behavioral taxonomy. The taxonomy has two major components - emotion and behavioral mitigation factors. Figure 2 contains our preliminary behavioral taxonomy. The taxonomy contained in Figure 2 is rather large and could potentially produce hundreds of actions that interact with emotion and other mitigation factors. Although some efforts have attempted to develop mathematical models that account for large numbers of behavioral factors, these models cannot be easily integrated into the virtual environment to provide a realistic and predictable behavioral response and, thus may potentially confound the type of training the VECTOR seeks to deliver. The complexity of implementation, the refinement of avatar behaviors based on complex emotional models, and the assessment of the validity of the probabilistic output of the model are beyond the scope ofthis effort. We have developed an alternative initial approach to modeling emotion, which is presented below.

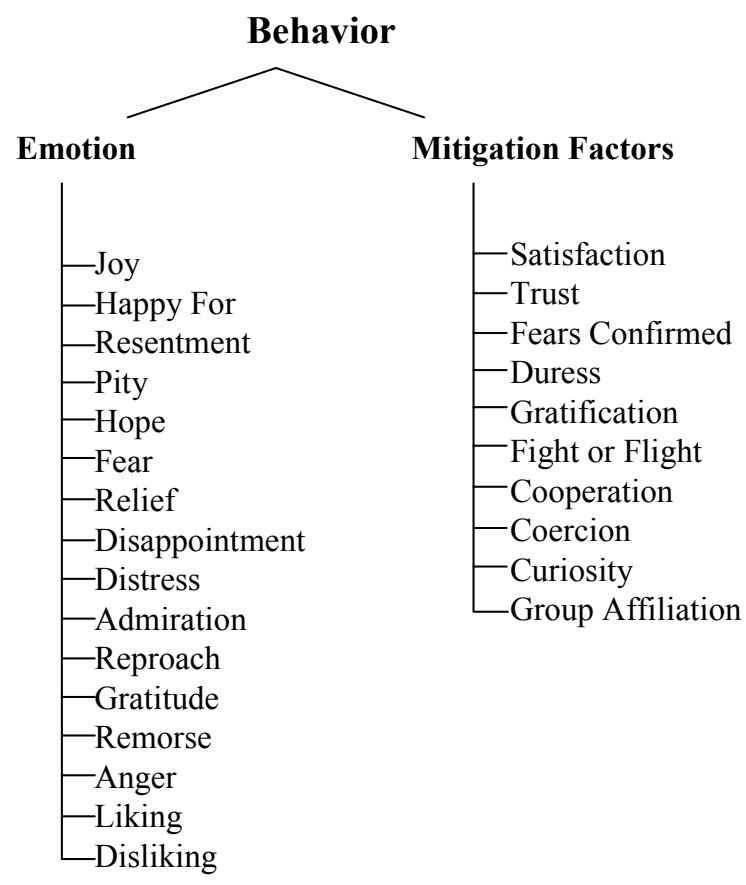

Figure 2: VECTOR NPC Behavior Taxonomy

\subsection{Preliminary Emotional Model}

In order to facilitate the implementation of the virtual environment, we are initially using a lower risk approach to attain a reasonable level of emotional model behavior. Our initial concept of the emotional model is to establish a set of variables that represent levels of each of six "basic" emotions. Commonly, this set includes happiness, sadness, anger, fear, disgust, and surprise (Velasquez 1997). For our purposes, we can choose to augment this set with other emotional variables (such as trust). Such additional variables can be chosen on the basis of their benefit to the realism of training, regardless of their actual theoretical validity.

The emotional variables are controlled by a set of (culture-specific) mappings that take output from the dialog manager, and from events in the environment and apply corresponding adjustments to one or more of these variables. The mechanism can be elaborated to include excitatory and inhibitory effects between emotional variables, as well as saturation levels and a decay curve.

The emotional state (i.e., vector of emotional variables) influences the triggering, priority, and execution of cognitive tasks within the model. A combination of anger and fear, for example, in conjunction with an environmental stimulus (a gun being drawn) might trigger a selfdefense task, involving a violent response. Instead of selecting anger or fear, the model might select a lesser reaction to the appearance of the weapon (such as a verbal response, or a decision to cooperate).

Figure 3 provides a listing of the 7 emotional factors that are used in the current NPC cognitive model for all Ingenious Entities. All of the emotional factors have an ordinal scale from 0 to 10 , with 5 being the median value. Figure 3 also shows the interpretation of the scale values for each factor. Note that high values (i.e., 10) for the happiness and trust factors are good from the Trainee's perspective while low values (i.e., 0) are ideal for the sadness, anger, fear, disgust, and surprise factors.

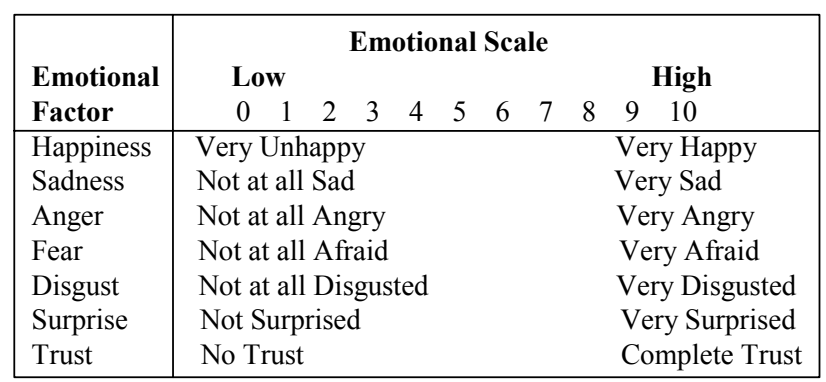

Figure 3: VECTOR NPC Emotional Scales

Indigenous Entities do not start the simulation with the same emotional state. This variation is done to better model the anticipated differences between entities based on factors such as gender, age, social status, and political affiliation. Figure 4 provides our preliminary initialization values for the emotional state of all Indigenous Entities. The male Civilian Entity represents the most natural of all entities and has initial values of 5 for all emotional factors except trust Trust will have a significant impact on the degree to which an entity will supply useful information. For this reason the trust factor are set to 0 for male Civilian Entities.

In the virtual environment, trust must be earned, and interactions with trusting Civilian men are the predominant 


\begin{tabular}{|c|c|c|c|c|c|c|}
\hline \multirow[b]{2}{*}{ Factor } & \multicolumn{6}{|c|}{ Entity } \\
\hline & $\begin{array}{c}\text { Civilian } \\
\text { Man }\end{array}$ & $\begin{array}{l}\text { Civilian } \\
\text { Woman }\end{array}$ & $\begin{array}{c}\text { Civilian } \\
\text { Child }\end{array}$ & Imam & $\begin{array}{c}\text { Local } \\
\text { Authority }\end{array}$ & $\begin{array}{c}\begin{array}{c}\text { Person } \\
\text { of } \\
\text { Interest }\end{array} \\
\end{array}$ \\
\hline Happiness & 5 & 5 & 7 & 5 & 5 & 2 \\
\hline Sadness & 5 & 5 & 3 & 5 & 5 & 5 \\
\hline Anger & 5 & 3 & 3 & 5 & 5 & 7 \\
\hline Fear & 5 & 7 & 3 & 3 & 3 & 7 \\
\hline Disgust & 5 & 5 & 0 & 7 & 5 & 7 \\
\hline Surprise & 5 & 5 & 5 & 5 & 5 & 5 \\
\hline Trust & 0 & 0 & 5 & 0 & 5 & 0 \\
\hline
\end{tabular}

Figure 4: NPC Initial Emotion Values

way of collecting useful mission information. The exception to the 0 trust rule is the initial values for Civilian child and Local Authority Entities. We presume that child entities will have some level of inherent trust purely due to childhood innocence and curiosity. Local Authorities are assumed to have been trained by U.S. forces (reflecting the present mission in Iraq) and have had some level of indoctrination. Since the Local Authorities have been returned to their native environment and are no longer under the direct supervision of U.S. forces, we believe that trust will have decayed from the level attained during training. Local Authorities have neutral values for all other factors except fear. In future versions of VECTOR, local authorities will be armed and be in a position of power, and, thus, will tend to be less fearful than other entities.

Female Civilian Entities have identical values to Civilian men with two exceptions. We will assume for gender reasons that the Civilian women will have a slightly lower anger level (i.e., predisposition for violent behavior) and a slightly higher fear level.

Civilian child entities are, in general, happier, less sad, less angry, and have a lower fear level at the start of a simulation. This again is presumed to be an artifact of childhood innocence. We feel that disgust is a function of political and military views and affiliations and therefore is less applicable to child entities.

The initial values for the Imam, or mosque leader, is neutral (i.e., 5) for all factors except fear, disgust, and trust. The Imam will have a lower value for fear (clerics with strong convictions tend to be less fearful) but a higher value for disgust (due to strong political and religious views contrary to the presence of U.S. forces).

The initial values for the Person of Interest entities significantly diverge from those of the other entities. Persons of Interest entities have low levels of happiness, and higher levels of fear, anger, and disgust. Levels of sadness and surprise are neutral. The value for trust in Persons of Interest will always be 0 . Persons of Interest are the targets of the scenario and can never be made to trust the Trainee.

Figure 5 presents our current concept for the high-level architecture of the NPC emotional model. Connections in black are currently implemented, with future versions of VECTOR to include those connections/functions in blue. In this architecture, all Trainee actions and dialogs are evaluated against cultural and situational rules of behavior to determine a score on each of the emotional factors.

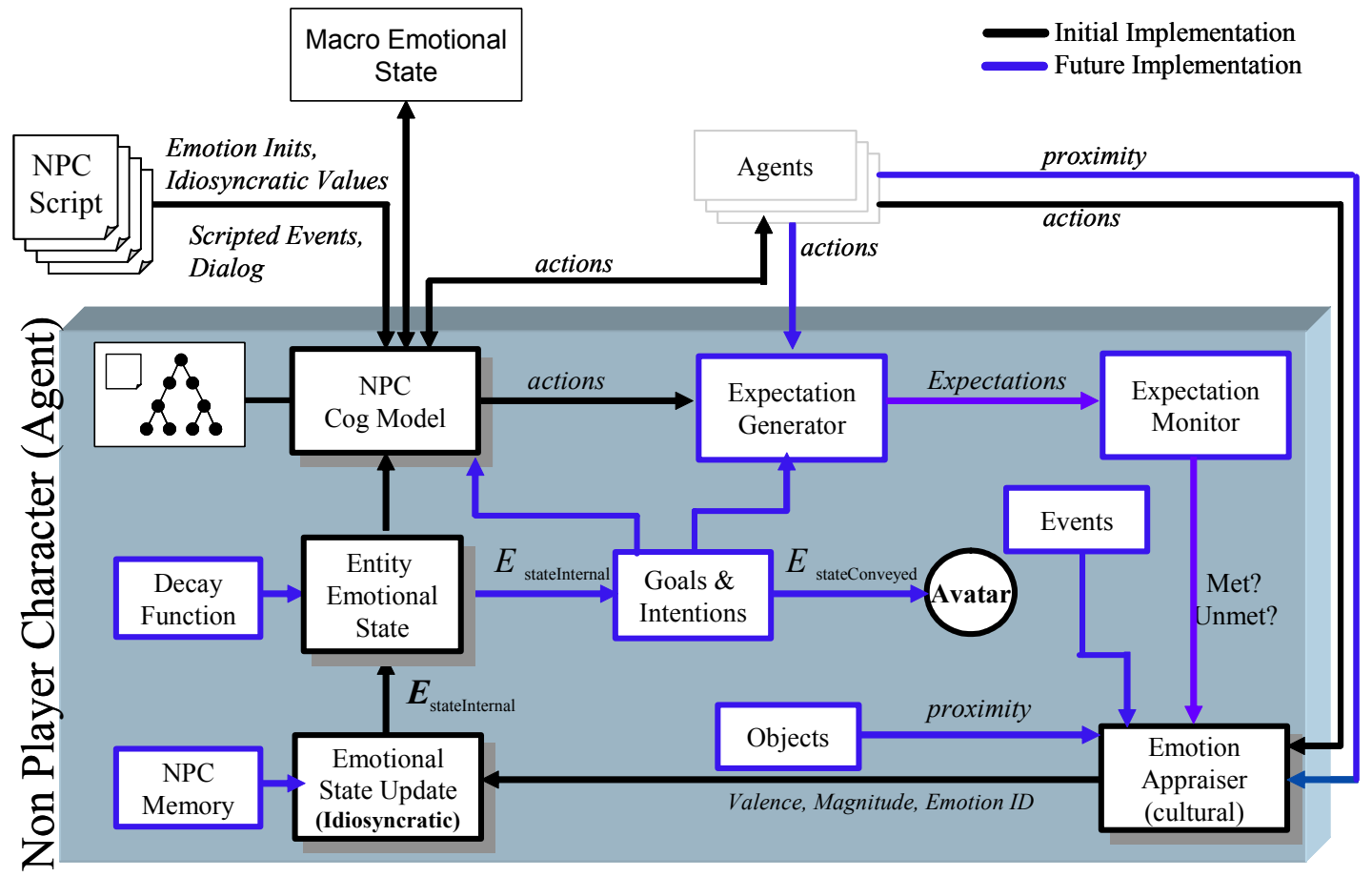

Figure 5: NPC Emotion Model Architecture 
Cultural rules are based on the appropriateness of the Trainee's action with respect to the current situation. For example, if the Trainee knocks on a door and female answers the door, the Trainee will be penalized if he or she tries to engage the female in conversation (other than asking for the male of the household). More specifically, this cultural violation may cause the female's value for fear and surprise to increase. Entity action and dialog generation (i.e., what the entity decides to do or say) is governed by the entities' internal emotional state.

All possible entity actions and dialogs are predefined and encoded as part of the NPC scripting capability of VECTOR. As previously mentioned, these script files are unique to each instantiated NPC and are analogous to an actor's script as part of a movie. The entity's internal decision logic first evaluates potential dialog and action choice according to situational rules, world states generated by hereto-fore trainee interactions, and based on gross emotional state. Once an action or dialog is selected, the specifics of the dialog or action will be determined by a finer level of evaluation of the emotional state. For example, if the entity's anger level is below 3 then the entity may choose to provide information to the Trainee. Additionally, if the entity's trust level is high and fear level is low the entity may provide useful intelligence information. However, if the entity's trust level is high but his fear level is also high (possibly due to the presence of a Person of Interest in his house) the entity may provide deceptive information.

\section{CONCLUSIONS}

We have developed in VECTOR a virtual training environment incorporating cognitive-model-controlled NPCs that facilitates the delivery of cultural-familiarization training. Through the use of a canonical cognitive model of NPC behaviors using the iGEN ${ }^{\mathrm{TM}}$ cognitive architecture and the utilization of a generic scripting language, cultural rules are encoded and mapped to scenario-specific NPC dialog and behaviors in order to provide a set of virtualNPC's with which the VECTOR trainee can interact. Additionally, the NPC model contains an emotional model that imparts the following capabilities:

- Modulation of NPC dialog and actions based on underlying trainee actions and dialog

- Mapping of emotional states to underlying trainee actions and dialog in order to measure cultural rule violations.

- Use of NPC emotional states to control grosslevel animation of NPC avatars conveyed emotional status.

Future areas of VECTOR development will include use of NPC emotional state to drive real-time facial animation of NPC avatars, deepening the VECTOR scenario to encode additional sets of cultural rule support, and adding additional features to the NPC emotion model.

\section{ACKNOWLEDGMENTS}

The VECTOR project was funded and sponsored by Dr. Bruce Knerr and Dr. Mike Singer through the U. S. Army Research Institute. In addition to the authors, the VECTOR system is being developed by Larry Rosenzweig, Vance Souders, and Jason Seip. Finally, domain expertise is being provided by Robert Morton and Mary Atwood from Sytex, Inc.

\section{REFERENCES}

Card, S., T. Moran., and A. Newell. 1983. The Psychology of Human-Computer Interaction. Hillsdale, NJ: Erlbaum.

Hayes-Roth, B. 1985. A blackboard architecture for control. Artificial Intelligence, 26, 251-321.

Izady, M. R. 1992. The Kurds: A concise handbook. Philadelphia, PA: Crane Russak.

Kreyenbroek, P., \& Allison, C. 1996. Kurdish culture and identity. New Jersey: Zed Books.

Ortony, A., Clore, G., and Collins, A., 1988. The Cognitive Structure of Emotions., Cambridge University Press.

Ruff, C. 1998. Exploring Islam. Washington Post, 13 May.

Strauss, C., \& Quinn, N. 1997. A cognitive theory of cultural meaning. Cambridge, England: Cambridge University Press.

Traindis, H.C. 1994. Culture and social behavior. New York, NY: McGraw-Hill.

Velasquez, J. D. 1997. Modeling emotions and other motivations in synthetic agents. Proceedings of AAAI-97. (pp 10-15). AAAI Press and The MIT Press.

Wise, C., Hannaman, D., Kozumplik, P., Ellen, F., and Leaver, B., 1998. Methods to improve cultural communication skills in Special Operations forces. ARI Contract Report 98-06. United States Army Research Institute for the Behavioral and Social Sciences.

Zachary, W.W., Ryder, J.M., Ross, L., and Weiland, M.Z., 1992. Intelligent computer-human interaction in realtime multi-tasking process control and monitoring systems. In Design for Manufacturability. ed. M. Helander and M. Nagamachi. New York: Taylor and Francis.

\section{AUTHOR BIOGRAPHIES}

THOMAS SANTARELLI is a Senior Cognitive Engineer at CHI Systems. His research interests include modeling and simulation of human cognitive and organizational behavior, decision support systems, and synthetic virtual actors. He has over 13 years experience in research into cognitive modeling, the study of human performance in military weapon systems, human-computer interface design and 
evaluation, and training system development. He has developed computational cognitive models using the iGENTM cognitive architecture for applications ranging from intelligent distributed team training and decision support systems to human performance modeling. He received his B.S. in Information Systems from Drexel University. His email address is <tsantarelli@chisystems.com> .

CHRIS MCCOLLUM is a cognitive engineer/social scientist with three years of applied professional experience and seven years of academic experience in cultural and psychological research/analysis. He received in Ph.D. in cultural anthropology from Duke University. While with CHI Systems, Dr. McCollum has designed methods for the collection and analysis of data on human and organizational performance, and has applied these methods in both field studies and experimental settings. Prior to joining CHI, Dr. McCollum held the position of Postdoctoral Fellow at Emory University, where he conducted and published research in cultural and personality theory. His email address is <cmccollum@chisystems.com>.

CHARLES BARBA is a Senior Human-System Engineer at CHI Systems with over 18 years of experience in the fields of industrial engineering, software development, and human-computer interaction. He has led research and software development efforts involving the study of human performance in advanced military systems ranging from human performance predictions in military systems to supervisory control of military satellite systems. He has a B.S. in Experimental Psychology from the University of Scranton and an M.E. in Industrial Engineering from Pennsylvania State University. His email address is <cbarba@chisystems.com>.

JOHN DEATON is a Managing Human Systems Engineer at CHI Systems. His Ph.D. is in Applied Experimental Psychology from The Catholic University of America, and has over 25 years experience working in the area of human performance, human factors engineering, decision support, cockpit automation, and training systems analysis. Dr. Deaton is also a retired Commander who was designated a Navy Aerospace Experimental Psychologist. He has participated in numerous DoD sponsored projects both during his Navy career and his current position at CHI Systems, including: the development of enhanced display formats for use in F-14D aircraft, research on the influence of $\mathrm{g}$ field on perceptual and cognitive performance, and the designing of decision support systems for emerging mechanical diagnostic systems for Naval rotorcraft. His email address is $<$ jdeaton@chisystems.com $>$. 